\title{
Cosmic distances from surface brightness fluctuations
}

\author{
John P. Blakeslee \\ Dominion Astrophysical Observatory, Herzberg Institute of Astrophysics, \\ National Research Council of Canada, Victoria, BC V9E 2E7, Canada \\ email: john.blakeslee@nrc-cnrc.gc.ca
}

\begin{abstract}
High spatial-resolution measurements of surface brightness fluctuations (SBFs) with the Hubble Space Telescope (HST) provide the most precise distances available to early-type galaxies beyond the Local Group. The observable SBF magnitude in a given bandpass is a basic property of any stellar system, corresponding to a ratio of the first and second moments of the stellar luminosity function. Calibration of the method has presented challenges, but we now have an excellent empirical determination of how the SBF observable varies with galaxy color in broad bandpasses at the red end of the optical spectrum, and we are working towards a similar calibration for HST's Wide-Field Camera 3 in the near-infrared wavelength range, where the SBF magnitudes are considerably brighter. From HST Advanced Camera for Surveys data, we have determined the relative distances of the Virgo and Fornax clusters to within a precision of $2 \%$, and resolved their internal structures. More recent measurements allow us to tie the Coma cluster, the standard of comparison for distant cluster studies, to the same precise distance scale. The SBF method can be calibrated in an absolute sense either empirically using Cepheids or theoretically based on stellar population models. The agreement between model and empirical zero points provides an independent confirmation of the Cepheid distance scale.
\end{abstract}

Keywords. galaxies: distances and redshifts, galaxies: clusters: individual (Virgo, Fornax, Coma), distance scale, large-scale structure of universe

\section{The SBF Method}

The measurement of the surface brightness fluctuations (SBFs) amplitude in high signal-to-noise images of the spheroidal components of galaxies is one of the most mature and accurate methods of determining galaxy distances. The SBF method was introduced and described in detail by Tonry \& Schneider (1988). In the years since, it has been refined and extensively applied for measuring distances to hundreds of galaxies (e.g., Tonry et al. 1990, 1997, 2001; Jensen et al. 1996, 1998, 2001; Ajhar et al. 1997, 2001; Blakeslee et al. 1999, 2009, 2010; Mieske et al. 2003, 2006; Mei et al. 2005a,b, 2007; Cantiello et al. 2005, 2007, 2011). Recent reviews of the SBF method are given by Blakeslee (2012) and Fritz (2012). Here, we briefly summarize the basic idea and provide a progress report on some current SBF projects.

Fig. 1 illustrates the observational properties of SBF data using a deep Hubble Space Telescope (HST) Advanced Camera for Surveys (ACS) image of the Virgo cluster galaxy M87. To measure the SBFs, the mean galaxy surface brightness must be carefully modeled and subtracted. Next, all contaminating sources down to some detection threshold must be masked. The image in Fig. 1 is unusually deep (the data were acquired for purposes other than measuring SBF magnitudes), and this allows the brightest red giant branch (RGB) stars to be detected directly (Bird et al. 2010). More typically, individual stars cannot be detected, but the statistical fluctuations in luminosity per resolution element can be measured from the image's power spectrum. The amplitude of the fluctuations 

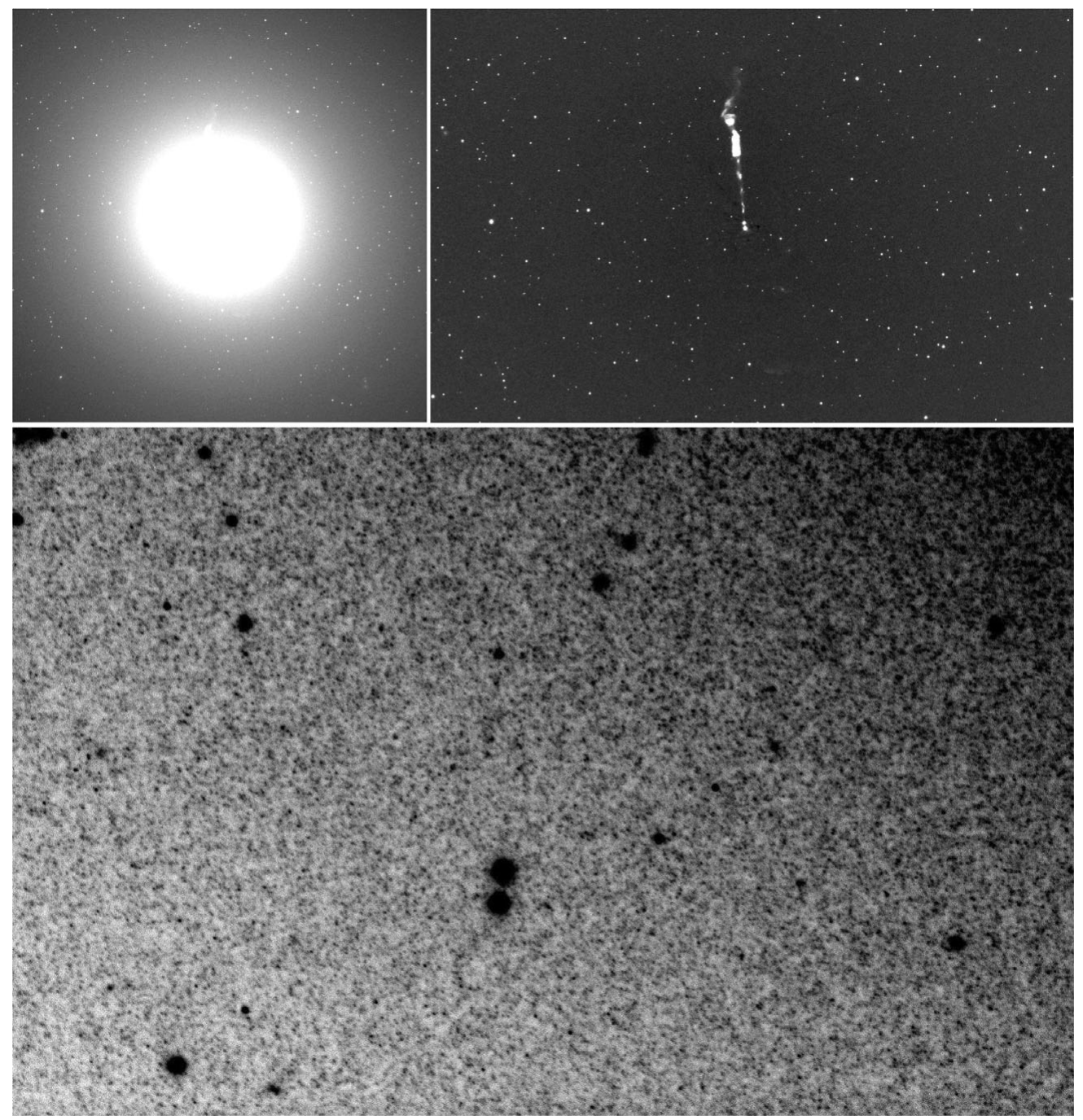

Figure 1. Surface brightness and fluctuations in M87, the central giant elliptical in the Virgo cluster of galaxies. (top left) $\sim 2^{\prime}$ portion of a stacked image in the $\mathrm{F} 814 \mathrm{~W}$ bandpass of the HST Advanced Camera for Surveys; the total exposure time is $73,800 \mathrm{~s}$. (top right) Zoom of the same image after subtracting a smooth model of the galaxy's surface brightness distribution, revealing the bright nuclear jet and numerous globular clusters. (bottom) Smaller region in the corner of the image: globular clusters and the bright tip of the stellar population are visible against a spatially fluctuating background. After normalization by the local surface brightness and calibration for the local population's color, the amplitude of these high signal-to-noise fluctuations gives a precise estimate of the distance.

is normalized by the local surface brightness and converted into the SBF magnitude, $\bar{m}$. The absolute SBF magnitude, $\bar{M}$, can be estimated from empirical or theoretical calibrations based on observable distance-independent stellar population properties, usually broadband color; the distance then follows.

The SBF properties of a galaxy vary with bandpass. Fig. 2 shows the predicted dependence of $\bar{M}$ on $\left(g_{475}-z_{850}\right)$ color for composite models with characteristics similar to the broad population of early-type galaxies (Blakeslee et al. 2001; see also Côté et al. 


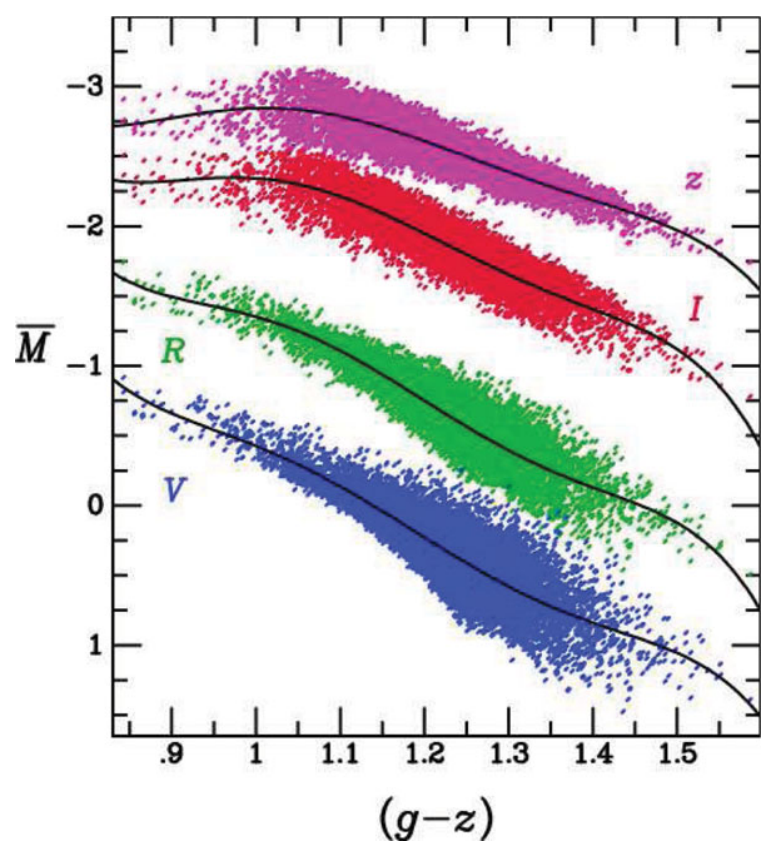

Figure 2. SBF magnitude in the $z$ (F850LP), $I, R$, and $V$ bandpasses as a function of galaxy $\left(g_{475}-z_{850}\right)$ color, based on the full range of early-type galaxy composite stellar population models in Blakeslee et al. (2001). The curves are simple polynomial fits to guide the eye. The SBF magnitude is considerably brighter in the redder passbands, and the dependence on population color is less steep overall. However, at bluer colors, the slope of the relation is predicted to change more (flattening out) in the redder passbands; the scatter also increases.

2004). Because the fluctuations are dominated by RGB stars in such galaxies, the color of the SBFs (differences in $\bar{M}$ ) is quite red, and the required exposure times are much shorter for redder bands. These models predict that the $I$-band SBF relation has roughly constant scatter with color, while the scatter decreases in $z$, but increases in $V$ and $R$, for models with colors typical of giant elliptical galaxies. The relations also exhibit differing amounts of curvature. Thus, it may be that $R$ would be a better choice for samples of blue dwarf galaxies in nearby groups (e.g., Jerjen et al. 2001; Dunn \& Jerjen 2006), while the more commonly used $I$ and $z$ bands are better for a broader range of galaxies and distances (see other references above). We discuss near-infrared (near-IR) SBF observations in Section 4.

\section{SBF Distances in Fornax and Virgo}

The ACS Virgo and Fornax Cluster Surveys (ACSVCS: Côté et al. 2004; ACSFCS: Jordán et al. 2007) are ambitious surveys with HST/ACS in the F475W $\left(g_{475}\right)$ and F850LP $\left(z_{850}\right)$ bandpasses, targeting 100 and 43 early-type galaxies, respectively. The goals of these surveys include detailed, high-resolution studies of galaxy structure, of the properties of thousands of globular clusters in the program galaxies, and of the 3D galaxy distributions within the clusters as determined by SBF distance measurements. Mei et al. (2005a) describe the SBF data reduction procedures implemented to deal with the unique properties of the ACS data. Analysis and results for the spatial structure of the Virgo and Fornax clusters are presented in Mei et al. (2007) and Blakeslee et al. (2009). With typical measurement errors of 0.07 mag in distance modulus, the cluster 

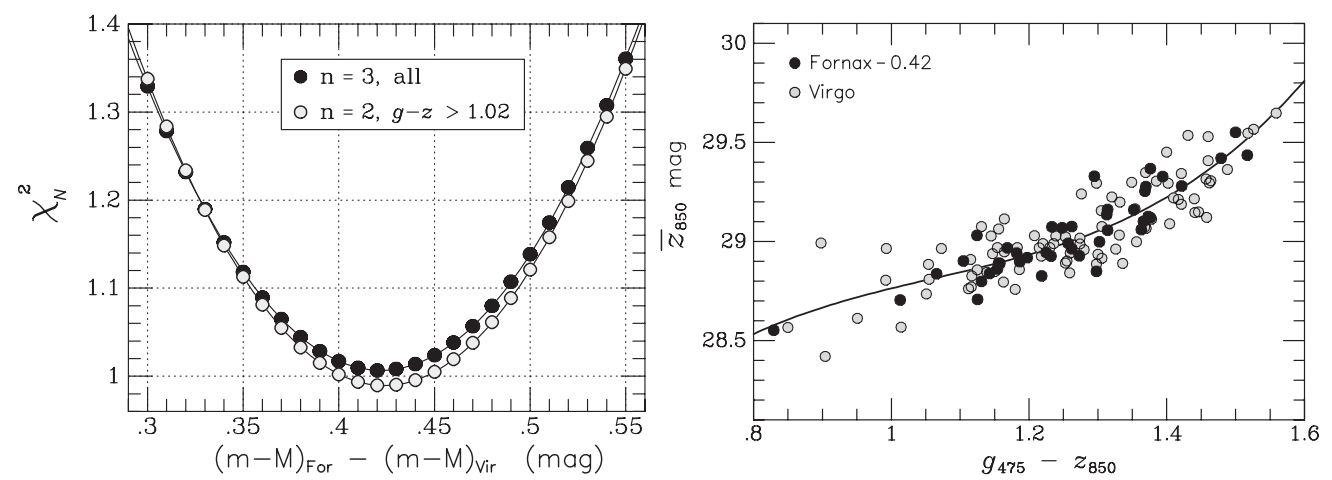

Figure 3. Combined SBF $\bar{z}_{850}$ versus $\left(g_{475}-z_{850}\right)$ color calibration for the ACS Virgo and Fornax Cluster Survey galaxies. (left) Reduced $\chi^{2}$ as a function of the relative Fornax-Virgo distance modulus; the same relation between SBF $z$ magnitude and $\left(g_{475}-z_{850}\right)$ color was used in both clusters. The black points include all galaxies in the fit and use a third-order polynomial for the stellar population calibration. The light-grey points show the results for a quadratic polynomial using only galaxies redder than $\left(g_{475}-z_{850}\right)=1.02$ mag. The $\chi^{2}$ computation includes an intrinsic scatter of $0.06 \mathrm{mag}$ in the relation (see Blakeslee et al. 2009 for details). (right) SBF calibration relation after shifting the Fornax galaxies nearer by the best-fitting relative distance modulus of $0.42 \pm 0.02 \mathrm{mag}$. The curve shows the cubic polynomial fit to the combined relation.

depth is clearly resolved in Virgo, but only marginally so for the more compact Fornax cluster. The interested reader should consult the above works for details.

Fig. 3 shows the SBF versus $\left(g_{475}-z_{850}\right)$ calibration for the ACS $z$-band SBF method derived simultaneously for the ACSVCS and ACSFCS data. The left panel shows how the reduced $\chi^{2}$ for the calibration relation varies for a range of offsets between the Fornax and Virgo distance moduli. The minimum $\chi^{2}$ is attained for a relative distance modulus of $0.42 \pm 0.03 \mathrm{mag}$, indicating that the Fornax cluster is $21 \%$ more distant than Virgo (the uncertainty also includes a correction for the maximum allowable age difference between the galaxies in both clusters). Accounting for measurement error and cluster depths, the intrinsic scatter in the method for galaxies with $\left(g_{475}-z_{850}\right)>1 \mathrm{mag}$ is only $0.06 \mathrm{mag}$, i.e., this is the scatter in $\bar{M}_{z}$ at a given $\left(g_{475}-z_{850}\right)$ color. This is approximately half the intrinsic scatter in Type Ia supernovae distances (e.g., Jha et al. 2007), making the $z$-band SBF method for early-type galaxies one of the most precise of all extragalactic distance indicators.

The zero point of the method is based on ground-based SBF measurements to the bulges of spiral galaxies with Cepheid-based distances (see Tonry et al. 2000, their Appendix B; Mei et al. 2005b; Blakeslee et al. 2010, their Appendix A). For a variety of reasons, these galaxies are not ideal for application of the SBF method. However, as shown in Fig. 4, the zero point predicted by the Teramo/SPOT models (Raimondo et al. 2005; Raimondo 2009) agrees very closely with the empirical value; this provides a useful, independent confirmation of the empirical calibration, including the Cepheid-based distance scale itself.

\section{SBF Distances to Coma and Beyond}

As the richest galaxy cluster within $c z<10,000 \mathrm{~km} \mathrm{~s}^{-1}$, the Coma cluster serves as the standard of comparison for diverse studies of galaxy properties and scaling relations. An accurate distance to the Coma core is essential for comparing its galaxy luminosities and sizes to nearby galaxies in Virgo and Fornax, while an accurate peculiar velocity is 


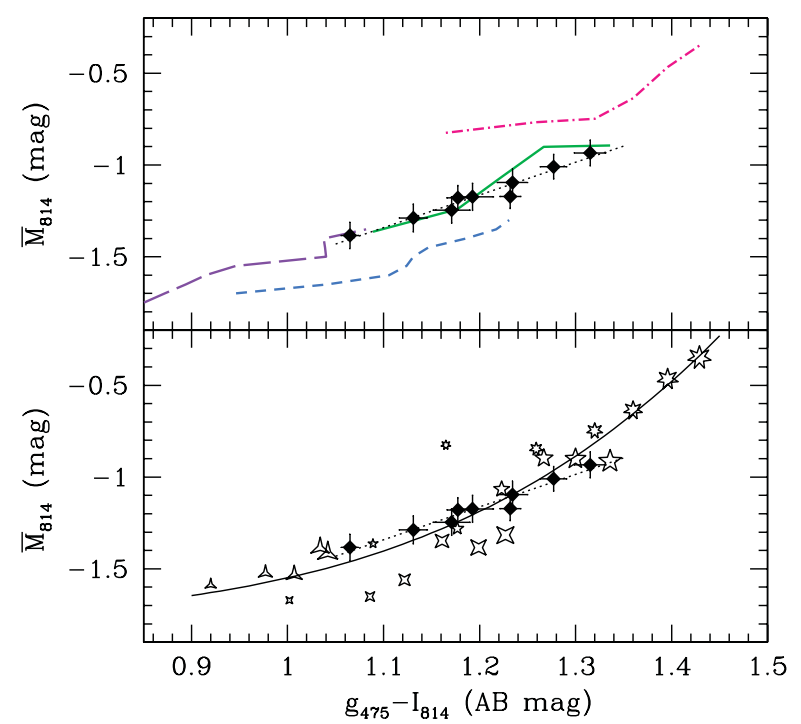

Figure 4. SBF as a primary and a secondary distance indicator: comparison of empirical and theoretical calibrations of the SBF method. (top) Predicted curves of F814W SBF magnitude as a function of $(g-I)$ color for Teramo/SPoT models of various metallicities with ages ranging from 3 to 14 Gyr. Black diamonds: Data for nine Fornax cluster ellipticals, assuming the $z$-band SBF distances, along with the empirical linear relation (dotted line). The solar-metallicity model predictions (solid green curve) coincide well with the observations, without any adjustment in zero point. (bottom) As for the top panel, but the individual models are represented by discrete points (see Blakeslee et al. 2010 for details). The curve is a cubic polynomial fit to the plotted models; within the color range of the data points, the relation appears linear, but the models predict increased curvature beyond this range.

needed for anchoring high-redshift cluster evolution studies. At $d \approx 100 \mathrm{Mpc}$, Coma is likely beyond the reach of accurate Cepheid measurements, and well beyond detection of the tip of the RGB, the other extragalactic methods with similar precision to SBFs. Even if Cepheids could be measured in a spiral within the Coma region, experience dictates that Cepheid distances to clusters must be carefully verified by high-quality distance measurements to the dominant early-type population.

Thus, we undertook to obtain precise SBF distances to NGC 4874 and NGC 4889, the two dominant ellipticals in the Coma core, using the well-calibrated F814W bandpass of HST/ACS. Fig. 5 shows the stacked four-orbit images of each galaxy. We emphasize that the goal is not to measure $\mathrm{H}_{0}$, which is increasingly well constrained by many different methods (e.g., Freedman \& Madore 2010; Riess et al. 2011). In fact, Biscardi et al. (2008) used the SBF method as a theoretically calibrated primary distance indicator out to $\sim 100 \mathrm{Mpc}$ and derived a Hubble constant $\mathrm{H}_{0}=76 \pm 6 \mathrm{~km} \mathrm{~s}^{-1} \mathrm{Mpc}^{-1}$, very close to the best Cepheid-based estimates. Rather, the main goals include obtaining precise relative distances (to $\sim 3 \%$ ) between Coma and the nearby Virgo and Fornax clusters, which already have excellent SBF data, and constraining the peculiar velocity of the Coma cluster in order to improve its tie to the Hubble flow and enable more accurate comparisons to high-redshift clusters. Our preliminary measurements place both galaxies at identical distances very near $100 \mathrm{Mpc}$. We are finalizing our estimates of the systematic uncertainties, mainly from the point-spread-function (PSF) normalization. We expect submission of the final results for publication in the immediate future. 


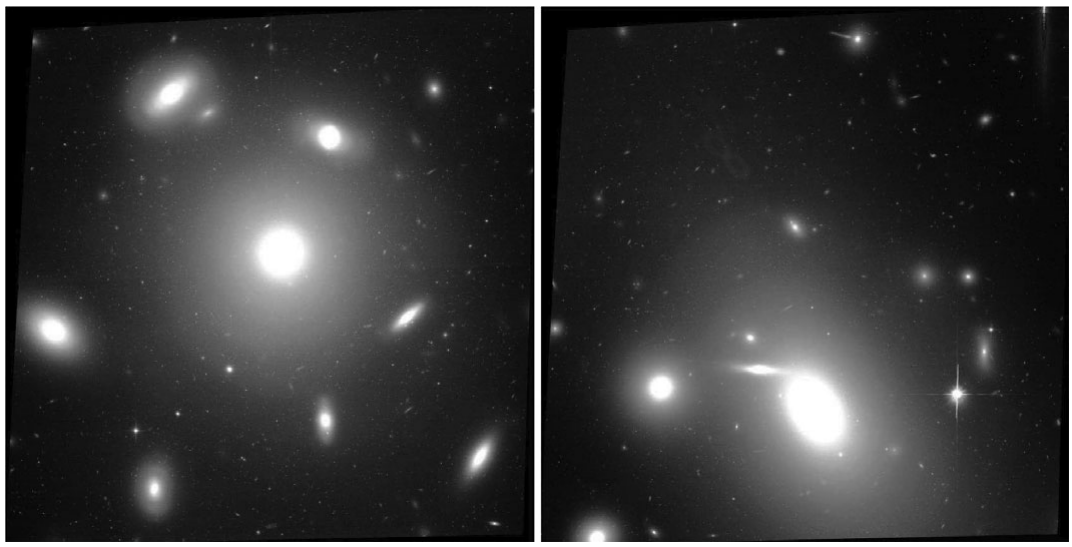

Figure 5. HST/ACS images of the two central giant ellipticals in the Coma cluster: NGC 4874 (left) and NGC 4889 (right). The exposures are four orbits each in F814W ( $I$ band) and allow Coma to be placed accurately on the same distance scale as the Virgo and Fornax clusters. Although the SBF magnitudes are brighter in the F850LP $(z)$ bandpass, the higher throughput of the broad F $814 \mathrm{~W}$ bandpass makes it more efficient for SBF measurements at these distances.

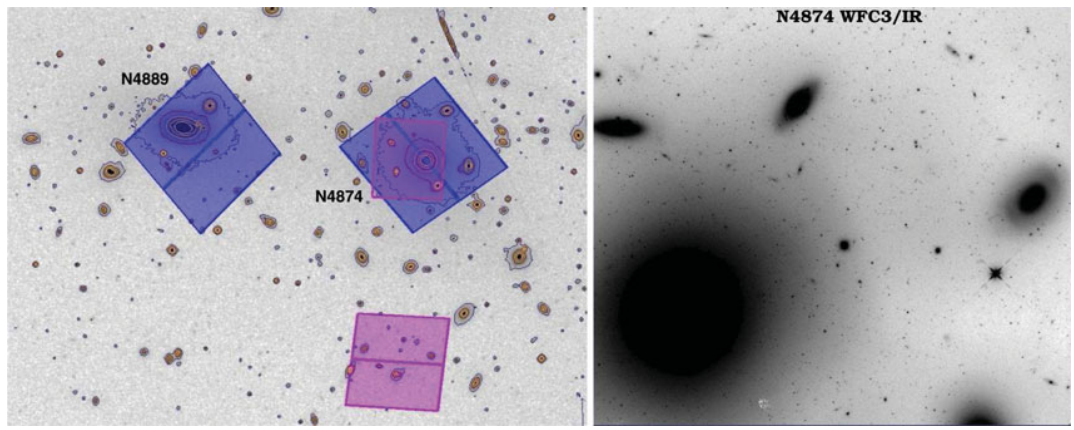

Figure 6. Geometry for the HST Coma cluster observations. (left) Arrangement of the primary ACS (blue parallelograms around NGC 4874 and NGC 4889) and parallel Wide-Field Camera 3 (WFC3; pink parallelograms) fields of view (FoVs) in the two HST pointings. The smaller WFC3 FoV represents the near-IR channel, which was used to observe NGC 4874 during the primary NGC 4889 observations; this is possible because of the fortuitous $\sim 5^{\prime}$ separation between these two central galaxies. Unfortunately, the opposite orientation was not possible during the relevant HST cycle; thus, no WFC3/IR observations were obtained of NGC 4889. Instead, the WFC3 optical channel (larger pink outline) was used to image a blank field in the Coma core. (right) WFC3/IR F160W image of NGC 4874.

\section{SBFs at Near-IR Wavelengths}

The installation of ACS on HST produced a renaissance in the optical SBF method: the method has been applied to ten times as many galaxies with ACS as with the WideField and Planetary Camera 2, and with stunning results, only briefly hinted at above. However, SBF magnitudes are much brighter in the near-IR because the main contributors in early-type galaxies are RGB stars: typical optical-IR colors are $\left(\overline{m_{I}}-\overline{m_{H}}\right) \approx 3.2$ and $\left(\overline{m_{I}}-\overline{m_{K}}\right) \approx 4 \mathrm{mag}$ (Jensen et al. 1998, 2003). This makes the near-IR regime very attractive for application of the SBF method, but the bright sky background and small formats of earlier IR detectors made such measurements extremely challenging from the ground. Sky background is much less of an issue from space, and Jensen et al. (2001, 2003) measured SBF magnitudes for 65 galaxies out to $130 \mathrm{Mpc}$ with the NICMOS 


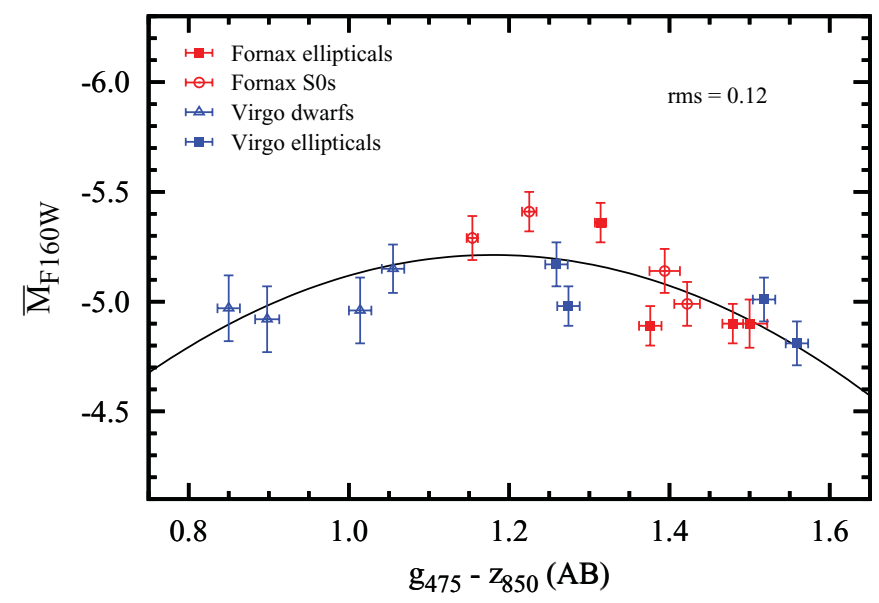

Figure 7. Preliminary empirical calibration of the SBF method for the HST WFC3/IR F160W bandpass. The absolute F160W SBF magnitudes are derived from the known optical SBF distances for these 16 Virgo and Fornax cluster galaxies. The plotted $\left(g_{475}-z_{850}\right)$ colors are on the AB magnitude system as published in the ACS Virgo and Fornax Cluster Survey papers, while the F160W SBF magnitudes are on the Vega system for comparison with previous IR SBF work with NICMOS. Interestingly, the bluest and reddest galaxies have very similar mean SBF magnitudes in this bandpass, while the SBF magnitude appears brighter for galaxies at intermediate color. This may be due in part to the selection of galaxies without signs of recent star formation at the blue end of the relation, where variations in age and metallicity are not degenerate in this plane. The scatter about the plotted quadratic curve is larger than the observational error and suggests an intrinsic scatter of $\sim 0.1 \mathrm{mag}$ in this relation.

F160W bandpass. The results were impressive, demonstrating the great promise of the near-IR SBF method, but the analysis was hindered by the NICMOS detector characteristics, especially the cosmic-ray persistence, which resulted in significant systematic uncertainties.

The launch of WFC3 with its large-format IR channel and improved cosmetics, has now opened a new era for the near-IR SBF method, similar to the effect of ACS in the optical. In fact, these are overlapping eras, since both ACS and WFC3 can be used simultaneously for SBF measurements. Fig. 6 illustrates the geometry for the HST Coma cluster observations discussed above. Because of the lucky $\sim 5^{\prime}$ separation between the two galaxies, it was possible to image NGC 4874 in parallel with WFC3/IR during the primary ACS observations of NGC 4889 (however, the reverse orientation was not schedulable during that observation cycle). The right panel of Fig. 6 shows the resulting high-quality 'free' F160W (similar to $H$ band) image of NGC 4874 .

To realize the full potential of WFC3/IR for SBF measurements, the stellar population dependence and intrinsic scatter in select passbands need to be quantified with the same precision as for the ACS F814W and F850LP filters. We are carrying out a program to calibrate the SBF method for the F110W $(J)$ and F160W $(H)$ bandpasses of WFC3/IR using observations of 16 Virgo and Fornax cluster galaxies that already have good ACS SBF distance measurements. Despite being only half an orbit in each filter, the SBF signal is extremely strong. Cho et al. (this volume) present a detailed status report on this project and comparisons to model predictions. Fig. 7 shows the preliminary calibration. The results suggest that the degree of curvature in the SBF-color relations from Fig. 2 increases in the near-IR, so that the bluest early-type galaxies exhibit similar SBF amplitudes as the reddest ones, and the brightest SBF magnitude occurs near $\left(g_{475}-\right.$ $\left.z_{850}\right) \approx 1.2 \mathrm{mag}$. We note that galaxies at the bluer colors may have a diversity of ages 
and metallicities, and the selection for this project was limited to those without obvious recent star formation. Overall, the intrinsic scatter appears to be $\sim 0.1$ mag or less; we are still assessing the observational errors due to PSF modeling and other effects. Once the calibration is finalized, the strong IR SBF signal, combined with the high throughput and wide field of WFC3/IR, should enable SBF distance measurements for suitable galaxies observed in these IR passbands to distances well out into the Hubble flow.

\section{Acknowledgements}

I thank the conference organizers for the invitation and my collaborators for the contributions to the above work, especially Hyejeon Cho and Joe Jensen for their work on the Coma and WFC3/IR data, and Michele Cantiello for Figure 4.

\section{References}

Ajhar, E. A., Lauer, T. R., Tonry, J. L., et al. 1997, AJ, 114, 626

Ajhar, E. A., Tonry, J. L., Blakeslee, J. P., Riess, A. G., \& Schmidt, B. P. 2001, ApJ, 559, 584

Bird, S., Harris, W. E., Blakeslee, J. P., \& Flynn, C. 2010, A\& A, 524, A71

Biscardi, I., Raimondo, G., Cantiello, M., \& Brocato, E. 2008, ApJ, 678, 168

Blakeslee, J. P. 2012, Ap\&SSS, 341, 179

Blakeslee, J. P., Cantiello, M., Mei, S., et al. 2010, ApJ, 724, 657

Blakeslee, J. P., Davis, M., Tonry, J. L., Dressler, A., \& Ajhar, E. A. 1999, ApJ, 527, L73

Blakeslee, J. P., Jordán, A., Mei, S., et al. 2009, ApJ, 694, 556

Blakeslee, J. P., Vazdekis, A., \& Ajhar, E. A. 2001, MNRAS, 320, 193

Cantiello, M., Blakeslee, J. P., Raimondo, G., et al. 2005, ApJ, 634, 239

Cantiello, M., Blakeslee, J. P., Raimondo, G., et al. 2007, ApJ, 668, 130

Cantiello, M., Brocato, E., \& Capaccioli, M. 2011, A\& A, 534, A35

Côté, P., Blakeslee, J. P., Ferrarese, L., et al. 2004, ApJS, 153, 223

Dunn, L. P. \& Jerjen, H. 2006, AJ, 132, 1384

Freedman, W. L. \& Madore, B. F. 2010, ARA $\& A$, 48, 673

Fritz, A. 2012, Publ. Astron. Soc. Aus., 29, 489

Jensen, J. B., Luppino, G. A., \& Tonry, J. L. 1996, ApJ, 468, 519

Jensen, J. B., Tonry, J. L., \& Luppino, G. A. 1998, ApJ, 505, 111

Jensen, J. B., Tonry, J. L., Barris, B. J., et al. 2003, ApJ, 583, 712

Jensen, J. B., Tonry, J. L., Thompson, R. I., et al. 2001, ApJ, 550, 503

Jerjen, H., Rekola, R., Takalo, L., Coleman, M., \& Valtonen, M. 2001, A\&SA, 380, 90

Jha, S., Riess, A. G., \& Kirshner, R. P. 2007, ApJ, 659, 122

Jordán, A., Blakeslee, J. P., Côté, P., et al. 2007, ApJS, 169, 213

Mei, S., Blakeslee, J. P., Tonry, J. L., et al. 2005a, ApJS, 156, 113

Mei, S., Blakeslee, J. P., Tonry, J. L., et al. 2005b, ApJ, 625, 121

Mei, S., Blakeslee, J. P., Côté, P., et al. 2007, ApJ, 655, 144

Mieske, S. \& Hilker, M. 2003, A\&SA, 410, 445

Mieske, S., Hilker, M., \& Infante, L. 2006, A\& $A$, 458, 1013

Raimondo, G. 2009, ApJ, 700, 1247

Raimondo, G., Brocato, E., Cantiello, M., \& Capaccioli, M. 2005, AJ, 130, 2625

Riess, A. G., Macri, L., Casertano, S., et al. 2011, ApJ, 730, 119

Tonry, J. L., Ajhar, E. A., \& Luppino, G. A. 1990, AJ, 100, 1416

Tonry, J. L., Blakeslee, J. P., Ajhar, E. A., \& Dressler, A., 1997, ApJ, 475, 399

Tonry, J. L., Blakeslee, J. P., Ajhar, E. A., \& Dressler, A., 2000, ApJ, 530, 625

Tonry, J. L., Dressler, A., Blakeslee, J. P., et al. 2001, ApJ, 546, 681

Tonry, J. L. \& Schneider, D. P. 1988, AJ, 96, 807 